\title{
Norois
}

Environnement, aménagement, société

$234 \mid 2015$

Dynamique des milieux humides, éducation à l'environnement, pratiques festives, tourisme et patrimonialisation

\section{Christine Margetic, Michaël Bermond, Valérie Jousseaume, Maxime Marie (coord.), Atlas des campagnes de l'Ouest}

\section{Camille Hochedez}

\section{(2) OpenEdition Journals}

\author{
Édition électronique \\ URL : http://journals.openedition.org/norois/5597 \\ DOI : 10.4000/norois.5597 \\ ISBN : 978-2-7535-4218-1 \\ ISSN : $1760-8546$ \\ Éditeur \\ Presses universitaires de Rennes \\ Édition imprimée \\ Date de publication : 30 juin 2015 \\ Pagination : 97-99 \\ ISBN : 978-2-7535-4183-2 \\ ISSN : 0029-182X
}

Référence électronique

Camille Hochedez, « Christine Margetic, Michaël Bermond, Valérie Jousseaume, Maxime Marie (coord.), Atlas des campagnes de l'Ouest », Norois [En ligne], 234 | 2015, mis en ligne le 30 juin 2015, consulté le 23 septembre 2020. URL : http://journals.openedition.org/norois/5597 ; DOI : https:// doi.org/10.4000/norois.5597

Ce document a été généré automatiquement le 23 septembre 2020.

(c) Tous droits réservés 


\title{
Christine Margetic, Michaël Bermond, Valérie Jousseaume, Maxime Marie (coord.), Atlas des campagnes de l'Ouest
}

\author{
Camille Hochedez
}

\section{RÉFÉRENCE}

Christine MARgetic, Michaël Bermond, Valérie Jousseaume, Maxime MARIE (coord.) 2014, Atlas des campagnes de l'Ouest, Rennes, PUR, 304 p.

1 C'est un très beau livre que nous offrent les quelque 70 auteurs de l'Atlas des campagnes de l'Ouest: près de 300 pages richement illustrées dans un format inhabituel pour les Presses universitaires de Rennes, qui traduit l'ampleur de la tâche et l'ambition de ce collectif. Ce volume constitue un moyen original de présenter les résultats des recherches menées dans le cadre de plusieurs programmes de recherche sur l'Ouest et de les diffuser auprès des acteurs et des citoyens, reflétant la démarche de coconstruction du savoir entre chercheurs et acteurs adoptée par l'équipe. En cela, cet atlas se veut aussi un moyen de communication pédagogique et accessible.

2 Même si, par définition, l'essentiel des illustrations de l'atlas est constitué de cartes réalisées par des géographes, la teneur du propos de l'ouvrage se veut pluridisciplinaire: l'équipe réunit aussi des philosophes, juristes, sociologues et anthropologues, signe de la diversité des regards portés sur les campagnes de l'Ouest. En revanche, les multiples façons d'envisager ces campagnes s'inscrivent bel et bien dans la tradition des géographes ruralistes de l'Ouest, dont certaines grandes figures (Nicole Croix, Jean Renard, Armand Frémont, Robert Hérin et Jean-Baptiste Humeau) ont participé à l'écriture de cet ouvrage en introduisant chacune de ses parties. L'atlas adopte la dialectique classique "permanences/mutations » : il revendique à la fois les 
racines historiques des recherches de l'école rurale de l'Ouest et cherche à mettre au jour des dynamiques contemporaines. Les cinq parties de l'atlas sont en effet construites autour d'une même structuration qui exprime ces évolutions: "De l'enracinement aux nouveaux ancrages", «De la paroisse à la communauté de communes ", " Du village au lotissement », « Du modèle agricole intensif à l'AMAP » et "Des campagnes laborieuses aux nouveaux paradis verts". Il s'agit donc bien de montrer des espaces en mouvement: "Oui, l'ouest bouge!»(p.9) affirment les coordinateurs de l'ouvrage. Les campagnes sont aujourd'hui des espaces d'innovation, et plus seulement l'espace de la modernisation agricole et du progrès technique. Les planches illustrent comment les espaces ruraux s'adaptent, innovent et expérimentent pour répondre à de nouveaux besoins liés aux changements de leurs fonctions : besoins de services (logement, santé) en lien avec l'attractivité démographique, adaptation aux exigences environnementales dans l'urbanisme rural contemporain, nouveaux services de loisirs qui correspondent à l'imaginaire rural des citadins, pratiques et expérimentations artistiques et culturelles.

3 Signe de ces évolutions, l'entrée agricole n'est pas dominante dans cet atlas, mais constitue une problématique parmi d'autres qui mettent l'accent sur la fonction résidentielle des campagnes. Certes, une partie entière est dédiée à l'agriculture, mais elle combine observations classiques et analyses plus innovantes. Elle prolonge et actualise la réflexion des géographes de l'Ouest sur la modernisation des campagnes, mais insiste sur les innovations sociales et environnementales (AMAP, développement de la filière bois-énergie) ou encore sur le développement de l'agriculture biologique. C'est une manière de porter un regard renouvelé sur les relations villes-campagnes, à travers par exemple des planches sur les dispositifs de protection de terres ou paysages agricoles comme les PEAN (Périmètres de protection et de mise en valeur des Espaces Agricoles et Naturels périurbains) ou les ZAP (Zones Agricoles Protégées) : envisager les politiques de protection foncière revient à souligner les concurrences d'usage et l'importance paysagère de l'agriculture autant que sa fonction nourricière. Même quand le thème est relativement classique, son traitement cartographique (mode de représentation, échelle) est original et présente un réel apport. Par exemple la carte représentant les combinaisons d'usages agricoles des sols (p.177) est réalisée en divisant l'espace par des hexagones représentant une maille de $5 \mathrm{~km}$ de côté.

4 La richesse de l'illustration constitue le point fort de cet atlas : des cartes, évidemment, à différentes échelles. À l'échelle du Grand Ouest, c'est-à-dire l'espace allant «de l'estuaire de la Seine à celui de la Gironde» (p.9) (soient les Régions Haute-Normandie, Basse-Normandie, Bretagne, Pays de la Loire et Poitou-Charentes); mais aussi à l'échelle nationale, permettant des comparaisons avec le reste du territoire français; puis à l'échelle locale d'un territoire, d'une Région, d'un village, autant de changements scalaires qui permettent d'illustrer le propos par des études de cas dont certaines apportent des éléments d'analyse sur des lieux emblématiques de ces campagnes de l'Ouest (planche sur l'habitat à Camembert, ou sur les industries agro-alimentaires). À côté de ces cartes, l'atlas est illustré par des représentations plus originales: graphiques, photos, affiches, BDgraphie ou dessins originaux comme ceux d'olivier Lemire. La diversité des modes de représentation reflète la variété des sources utilisées: statistiques, enquêtes de terrain, presse régionale, littérature, cinéma, documents d'archive. L'analyse des formes d'habitat qui croise diverses sources (cartes, photographies, photographie aérienne...) est de ce point de vue très pédagogique. Cette variété des sources et des illustrations est couplée à une structuration efficace selon le 
principe de la double-page : pour une thématique, les illustrations sur la page de droite sont accompagnées d'un texte d'analyse en page de gauche, qui intègre des enquêtes de terrain. Ces allers-retours entre statistiques régionales et études de cas locales donnent chair aux analyses. L'atlas se veut donc thématique et non exhaustif, mais les choix thématiques auraient pu être davantage contextualisés dans l'introduction par rapport aux thématiques des programmes de recherche qui ont orienté celles de l'atlas. Cela aurait pu éclairer le lecteur sur les raisons de l'absence, par exemple, des thématiques environnementales, jamais traitées frontalement.

5 L'ouvrage soulève - en même temps qu'il cherche à répondre à - plusieurs questions. D'abord, celle de la définition de l'Ouest, qui est certes contrainte par le découpage des programmes PSDR, mais faire un atlas des campagnes de l'Ouest revient à supposer qu'elles présentent des spécificités qui permettent de les distinguer par rapport à d'autres régions. L'Ouest constituerait-il un "type » d'espace rural particulier? Si certaines dynamiques présentées sont communes aux espaces ruraux européens (périurbanisation, multifonctionnalité, etc.), d'autres sont en revanche plus originales et illustrent cette spécificité comme le poids de l'enseignement privé qui donne d'ailleurs lieu à des cartes particulièrement réussies sur le système scolaire breton, et à une analyse de l'école publique comme marqueur des espaces périurbains. On pourra en revanche regretter que certaines spécificités de l'Ouest (notamment la présence importante des espaces littoraux) ne soient pas représentées. La deuxième question soulevée est celle des valeurs et des représentations associées aux campagnes de l'Ouest. D'ailleurs, le titre de l'ouvrage préfère le terme de "campagnes " à celui d'espaces ruraux, signe de l'investissement de ce type d'espaces par des valeurs positives et par un imaginaire largement façonné par opposition à la ville. Ainsi, la définition des espaces ruraux est abordée dans cet atlas de manière statistique, en questionnant les seuils entre rural et urbain, mais aussi par les représentations comme en témoigne la planche sur les cartes postales régionales érotiques réalisée par $\mathrm{S}$. Valognes.

6 Cet atlas donne donc à voir la richesse et la diversité des campagnes de l'Ouest, mais aussi les disparités qui les traversent en termes de santé ou de revenus. Il est donc fidèle à la tradition de géographie sociale particulièrement développée au sein de l'UMR ESO (Espaces et Sociétés) dont sont issus bon nombre des contributeurs. L'ambition de cet atlas n'est-elle pas de donner à voir les tenants et les aboutissant des défis auxquels sont confrontées ces campagnes (vieillissement, accompagnement de l'installation en périurbain, protection des terres agricoles...), mais aussi de rendre visibles les inégalités en vue d'agir? Cet atlas est donc un geste scientifique autant que politique et citoyen. 